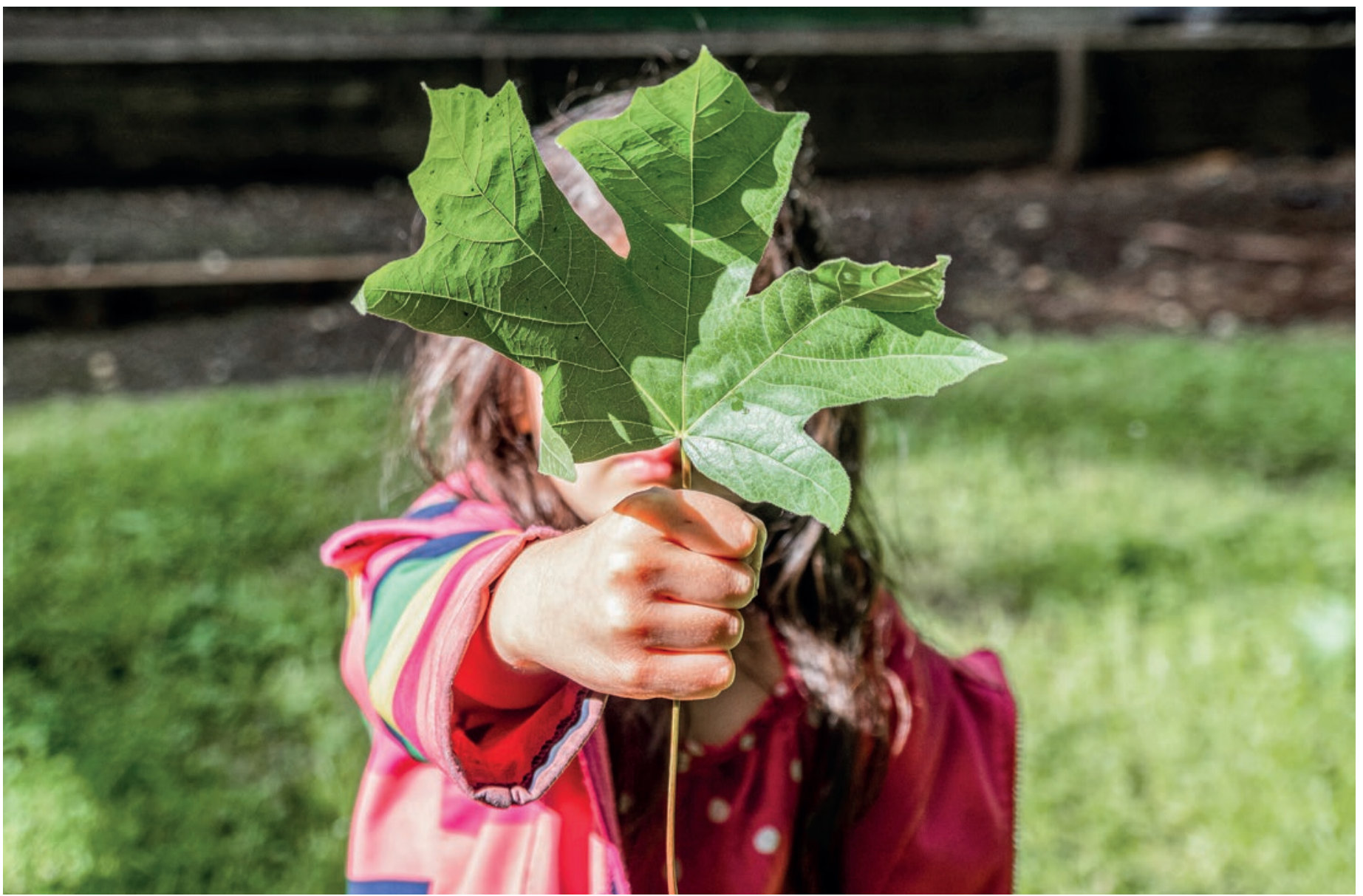

\title{
Für mehr Engagement der FMH in der Umweltpolitik
}

\section{Bernhard Aufdereggen}

Dr. med., Präsident Ärztinnen und Ärzte für Umweltschutz (AefU)

Die FMH als Repräsentantin der Ärzteschaft der Schweiz darf nicht nur standespolitische Interessen vertreten. Denn: Zu ihren zentralen Aufgaben gehört gemäss Statuten auch das Engagement für gesunde Umwelt- und Lebensbedingungen.

In unserer klinischen Tätigkeit sind wir als Ärztinnen und Ärzte neben der kurativ ausgerichteten Medizin oft mit Fragen der individuellen Prävention konfrontiert. Wir beraten unsere Patientinnen und Patienten und weisen sie darauf hin, mit dem Rauchen aufzuhören, ihre Ernährungsgewohnheiten zu ändern und sich mehr zu Fuss oder mit dem Velo zu bewegen. Bei der Aus- und Weiterbildung lernen wir in den Fächern Sozial- und Präventivmedizin oder neu im Public Health: Ungesunde Umwelt- und Lebensbedingungen können auch Krankheiten auslösen. Diese Umwelt- und Lebensbedingungen aber lassen sich meist nicht auf individueller Ebene beeinflussen. Dazu braucht es politische Diskussionen und Beschlüsse sowie Veränderungen auf gesellschaftlicher Ebene. 


\section{Prävention auf gesellschaftlicher und politischer Ebene}

Die Ärztinnen und Ärzte für Umweltschutz (AefU) bekämpfen seit über 30 Jahren krank machende Einflüsse durch die verschmutzte Umwelt auf individueller, aber vor allem auf der gesellschaftlichen Ebene. Wir beraten zwar auch einzelne Patientinnen und Patienten, die durch Umweltverschmutzungen erkranken. Um Krankheiten wegen Umweltnoxen vorsorglich zu verhindern oder zu bekämpfen, führt die politische Arbeit aber meist eher zum Ziel. Wir bauen folglich öffentlichen Druck auf, verfassen Stellungnahmen zu Umweltproblemen, diskutieren mit Politikerinnen und Politiker und der Verwaltung. Wir beziehen immer dann Position, wenn es darum geht, krank machende Umwelteinflüsse zu verhüten oder zu beseitigen.

\section{Die Rolle der Ärzteschaft}

Die FMH als einzige Vertreterin der gesamten Ärzteschaft der Schweiz äusserte sich in der Vergangenheit selten zu krank machenden Umwelt- und Lebensbedingungen. Das stört Studierende der Medizin sowie die Assistenz- und Oberärztinnen und -ärzte. Via Ärztekammer haben sie im Herbst 2020 von der FMH im Bereich Klima/Gesundheit mehr Engagement verlangt [1]. Auch vor den Abstimmungen über die Trinkwasserund Pestizid-Initiative sowie zum $\mathrm{CO}_{2}$-Gesetz wurde die FMH aufgefordert, Stellung zu beziehen. Solche

\section{Das Engagement für den Schutz vor Umwelt- gefahren bedeutet Prävention und ist direkt mit dem Arztberuf verbunden.}

Forderungen hat Yvonne Gilli, Präsidentin der FMH, in einem Editorial in der Schweizerischen Ärztezeitung vom 30. Juni 2021 zurückgewiesen und «für einen klaren Fokus der FMH auf standespolitische ärztliche Themen" plädiert [2]

Diese Haltung ist teilweise nachvollziehbar, steht die FMH doch aktuell an verschiedenen Fronten - u.a. im Bereich Tarifreform - unter Druck. Aber gerade in dieser Situation ist es für die Akzeptanz der Ärzteschaft in Gesellschaft und Politik von zentraler Bedeutung, sich (auch) für die Gesunderhaltung der Bevölkerung einzusetzen. Das setzt auch eine gesunde Umwelt voraus.

\section{Der Auftrag in den Statuten der FMH}

Bezüglich des fehlenden Auftrags der Mitglieder, den Gilli moniert, verweise ich auf Artikel 2 der FMH-Statu- ten «Die FMH bezweckt: a. Der Bevölkerung eine hoch stehende ärztliche Versorgung zu angemessenen Kosten zu gewährleisten, zur Gesundheitsförderung beizutragen und sich für die Erhaltung gesunder Umweltund Lebensbedingungen einzusetzen [3].» Die Statuten verlangen zwar den standespolitischen Ansatz. Sie fordern aber ebenso eine klare Position gegenüber krank machenden Umwelt- und Lebensbedingungen.

An wissenschaftlich aufgearbeiteten Beispielen von Umwelt- und Lebensbedingungen, die krank machen,

Wer, wenn nicht wir, können kompetent über die Auswirkungen einer verschmutzten Umwelt auf die Gesundheit berichten?

mangelt es nicht: Etwa die vielen Opfer des langjährigen Asbestgebrauchs oder die ungesunde Luftverschmutzung. Weitere zufällig ausgewählte Beispiele sind: der Auto- und Fluglärm, Blei im Boden, Quecksilber im Garten oder Benzidin auf alten Fabrikgeländen und in Deponien.

In allgemeiner Form auf die gesundheitlichen Gefahren dieser Umweltnoxen hinzuweisen, ist ein Schritt in die richtige Richtung. Konkret entscheiden aber in der Schweiz das Parlament und letztlich die Stimmberechtigten an der Urne die politischen Fragen, wie im vergangenen Juni bei den Abstimmungen über das $\mathrm{CO}_{2}$-Gesetz und die Pestizid-Initiativen. Es würde der FMH gut anstehen, sich bei solchen Abstimmungen klar zu positionieren. Sie würde dabei nicht an politischer Glaubwürdigkeit verlieren, im Gegenteil: Das Engagement der Ärzteschaft für den Schutz der Menschen vor gesundheitsgefährdenden Umweltgefahren bedeutet Prävention und ist somit direkt mit dem Arztberuf verbunden.

\section{Die Ärzteschaft und die Klimafrage}

Die Medizinstudentinnen und -studenten und die jüngeren Ärztinnen und Ärzte haben sich in den letzten Jahren zu Recht auf die gesundheitlichen Auswirkungen der Klimaveränderungen fokussiert. Die wissenschaftliche Basis dazu liefert der «Lancet Countdown on Health and Climate Change». Darin ziehen Wissenschaftlerinnen und Wissenschaftler von 35 akademischen Institutionen aus der ganzen Welt den Schluss: «Der Klimawandel ist die grösste globale Gesundheitsbedrohung, mit der die Welt im 21. Jahrhundert konfrontiert ist, aber er bietet auch die grösste Chance, die sozialen und ökologischen Bedingungen für Gesundheit neu zu definieren [4].»

Wer, wenn nicht wir Ärztinnen und Ärzte, können kompetent über die Auswirkungen einer verschmutz- 
ten Umwelt und eines veränderten Klimas auf die Gesundheit der Menschen berichten? Wer, wenn nicht wir, kann besser zur Änderung der Situation auffordern? Dies umso mehr, da die meisten Massnahmen zur Dämpfung des Klimawandels einen gesundheitlichen Zusatznutzen haben: Der Ausstieg aus der Verbrennung von Öl, Gas und Kohle (in Heizungen/

\section{Die meisten Massnahmen zur Dämpfung des Klimawandels haben einen gesundheitlichen Zusatznutzen.}

Verkehr/Industrie) führt zu weniger Luftverschmutzung und weniger Lärm und damit zu mehr Gesundheit. Die Veränderung der Essgewohnheiten mit dem Ziel einer verstärkt pflanzenbasierten Ernährung wirkt nicht nur positiv auf das Klima, sondern auch auf die Gesundheit durch weniger Herz-Kreislauf-Erkrankungen und weniger Krebs im Verdauungstrakt.

\section{Emissionen im Gesundheitssektor}

Nicht zu vernachlässigen ist auch der $\mathrm{CO}_{2}$-Ausstoss des schweizerischen Gesundheitssektors. Gemäss Schätzungen trägt er mit sechs bis sieben Prozent zu den Treibhausgas-Emissionen der Schweiz bei. In unserer Fachzeitschrift OEKOSKOP haben wir die gesundheitlichen Konsequenzen des Klimawandels aufgearbeitet [5]. Zusammen mit allen anderen Beteiligten im Gesundheitsbereich soll die Ärzteschaft eine Führungsrolle einnehmen und als Vorbild vor-

\section{Das Wichtigste in Kürze}

- Ungesunde Umwelt- und Lebensbedingungen sind gewichtige Auslöser von Leiden und Krankheiten.

- Um diese schädigenden Einflüsse einzudämmen, braucht es politische Diskussionen und Veränderungen auf gesamtgesellschaftlicher Ebene.

Ärztinnen und Ärzte für Umweltschutz

www.aefu.ch bernhard.aufdereggen[at] aefu.ch
- Die Ärztinnen und Ärzte für Umweltschutz sehen es als eine Aufgabe der FMH an, sich bei umweltpolitischen Themen klar zu positionieren und so zur präventiven Gesunderhaltung der Bevölkerung beizutragen. angehen [6]. Darum fordern die AefU eine Taskforce für Netto-Null- $\mathrm{CO}_{2}$-Emissionen im Gesundheitsbereich bis 2030.

Die Menschen - und v.a. die Kinder - werden es der Ärzteschaft danken, denn: «Das Leben jedes Kindes, das heute geboren wird, wird durch den Klimawandel tiefgreifend beeinflusst werden, da die Bevölkerung auf der ganzen Welt zunehmend mit extremen Wetterbedingungen, unsicherer Ernährung und Wasserversorgung, veränderten Mustern von Infektionskrankheiten und einer weniger sicheren Zukunft konfrontiert ist. Ohne schnelles Eingreifen wird dies die Gesundheit der Menschen in jeder Phase ihres Lebens bestimmen [7].»

\section{Bildnachweis}

Alan Rodriguez / Unsplash

\section{Literatur}

1 swimsa.ch/download/2426/MM_rzteschaft_und_Klimawandel_ DE_20201116_V01.00.pdf

2 Gilli Y. «Health in all policies» aber nicht "All policies in health». Schweiz Ärzteztg. 2021;102(26):868.

3 www.fmh.ch/files/pdf7/statuten-fmh.pdf, Hervorhebung durch den Autor.

4 Im Original: "Climate change is the greatest global health threat facing the world in the 21st century, but it is also the greatest opportunity to redefine the social and environmental determinants of health.» www.thelancet.com/article/ S0140-6736\%252820\%252932290-X/fulltext

5 www.aefu.ch/fileadmin/user_upload/aefu-data/b_documents/ oekoskop/Oekoskop_21_1_DS.pdf

6 www.aefu.ch/fileadmin/user upload/aefu-data/b documents/ oekoskop/Oekoskop_21_2_DS.pdf

7 www.lancetcountdown.org/2019-report/

\section{L'essentiel en bref}

- Les conditions environnementales et de vie malsaines sont des facteurs importants d'affections et de maladies.

- Afin d'enrayer ces influences néfastes, des discussions et des changements politiques sont nécessaires au niveau de la société dans son ensemble.

- Les Médecins en faveur de l'environnement considèrent qu'il est du devoir de la FMH de prendre clairement position sur les questions de politique environnementale et de contribuer ainsi au maintien préventif de la santé de la population. 